\title{
A Disturbance Suppression Micro-Newton Force Sensor Based on Shadow Method
}

This paper was downloaded from TechRxiv (https://www.techrxiv.org).

LICENSE

CC BY 4.0

SUBMISSION DATE / POSTED DATE

$12-01-2022$ / 14-01-2022

CITATION

Yang, Yong; Zhao, Meirong; Li, Dantong; Tao, Moran; Zhu, Chunyuan; Zheng, Yelong; et al. (2022): A Disturbance Suppression Micro-Newton Force Sensor Based on Shadow Method. TechRxiv. Preprint. https://doi.org/10.36227/techrxiv.18227783.v1

$\mathrm{DOI}$

10.36227/techrxiv.18227783.v1 


\title{
A Disturbance Suppression Micro-Newton Force Sensor Based on Shadow Method
}

\author{
Yong Yang, Meirong Zhao, Dantong Li, Moran Tao, Chunyuan Zhu, Yelong Zheng \\ and Yu Tian
}

\begin{abstract}
The precision of micro-force measurement is determined by the sensitivity of force sensors and the magnitude of environmental disturbances. Damping, a process that converts vibrational energy into heat, is one of the most effective methods of suppressing disturbances. Inspired by the shadow formed at a pond when water striders walked on the water, a bionic viscoelastic-polymer micro-force (VPMF) sensor with a high damping ratio based on the shadow method was developed. In the VPMF sensor, the surface of the polymer was deformed by the contact of a cylindrical flat punch when the sensor was subjected to a normal force. A shadow with a bright edge was formed due to the refraction that parallel light went through the deformed surface. The force was in proportion to the change of the shadow diameter. The sensor optimal sensitivity was $2.15 \mu \mathrm{N} / \mathrm{pixel}$ and the measurement range was $0.981 \mathrm{mN}$. The damping ratio of the VPMF sensor was 0.22 on account of viscoelasticity, which could suppress disturbances effectively. The VPMF sensor could reduce the influence of disturbances by about $96.23 \%$ compared to the cantilever. The present study suggests that the VPMF sensor is hopefully applied to the reliable measurement of micro force under complex environments.
\end{abstract}

Index Terms-Disturbance suppression, micro-force measurement, shadow method, viscoelastic polymer.

\section{INTRODUCTION}

$\mathrm{T}$ he precision measurement of micro force $(\mu \mathrm{N}$ magnitude and below) is desperately needed in the field of aerospace[1], material science[2], biomedicine[3], [4], bio-manipulation[5], [6]. For instance, reliable measurement of the micro thrust in the complex disturbance environments is the premise to achieve drag-free control that functions as the cornerstone for space-based gravitational wave detection satellites moving along a geodesic path [7]. In bionics

Manuscript received Month $x x, 2 x x x$; revised Month $x x, x x x x$; accepted Month $x, x x x x$. This work was supported by the National Key Research and Development Program of China (No 2020YFC2008703). (Corresponding author: Yelong Zheng, Yu Tian.)

Yong Yang, Meirong Zhao, Dantong Li, Moran Tao, Chunyuan Zhu, and Yelong Zheng are with the State Key Laboratory of Precision Measuring Technology and Instruments, Tianjin University, Tianjin, 300072, China.

(e-mail: 3013202058@tju.edu.cn; meirongzhao@tju.edu.cn; lidantong@tju.edu.cn; tmr5209@tju.edu.cn; zhuchunyuan@tju.edu.cn; zhengyelongby@tju.edu.cn).

Yu Tian is with the State Key Laboratory of Tribology, Department of Mechanical Engineering, Tsinghua University, Beijing 100084, China (e-mail: tianyu@tsinghua.edu.cn). engineering, in-situ and accurate characterization of biological mechanics is indispensable to the design of bionic robots, such as designing the bionic water strider robot [8]-[10]. However, it is still a formidable challenge to measure these micro forces precisely.

The precision of micro-force measurement is determined by the sensitivity of the force sensor and the magnitude of environmental disturbances. The development of high sensitivity micro-force sensors, such as piezoelectric and capacitive sensors, is always the hotspot of research. Persano et al. [11] designed a high-performance piezoelectric device based on aligned arrangements of nanofibers of poly, whose resolution was $3.6 \mu \mathrm{N}$. Micro-force sensors based on optical principles [12]-[14] have a resolution as high as piconewton, such as atomic force microscopy (AFM) and optical tweezers which are widely used in materials science and biomedicine. However, the above sensors generally have the weakness of poor anti-disturbance ability.

The common micro-force measurement methods, measuring the deformation of the elastic element, require smaller stiffness to achieve better resolution. However, the system damping ratio is generally small under this condition. For example, the damping ratio of metal cantilever beams was less than 0.01 in the elastic range [15]. As a result, the influences of airflow disturbance and ground vibration cannot be avoided effectively accounting for poor measurement precision. Currently, researchers are devoted to the study of how to suppress environmental disturbances to improve precision. Luo et al. [16] measured the Newtonian gravitational constant, G, having the smallest uncertainty reported until now. Their apparatus was located in the passive thermal room situated in a cave laboratory to reduce the influence of temperature and vibration. Sun et al. [17] built two identical devices using the differential method to reduce the effect of vibration. The relative uncertainty was less than $4 \%$, but it was quite hard to fabricate two identical elastic elements. Kazimierski et al. [18] proposed an implementable denoising method based on a local statistic of the measured data's spectrum for efficient retrieval of the force signal encountered in micro-milling processes. However, the filtering algorithm would inevitably change the characteristics of the original signal. The vibration isolation system is also an effective method, such as the negative stiffness structure[19] whose stiffness can be linearly adjusted by changing the coil current, but it was hard to avoid the influence of airflow disturbance. The resolution of existing micro-force measurement systems can reach $\mu \mathrm{N}$ magnitude only when the control of environmental disturbances requirements is 


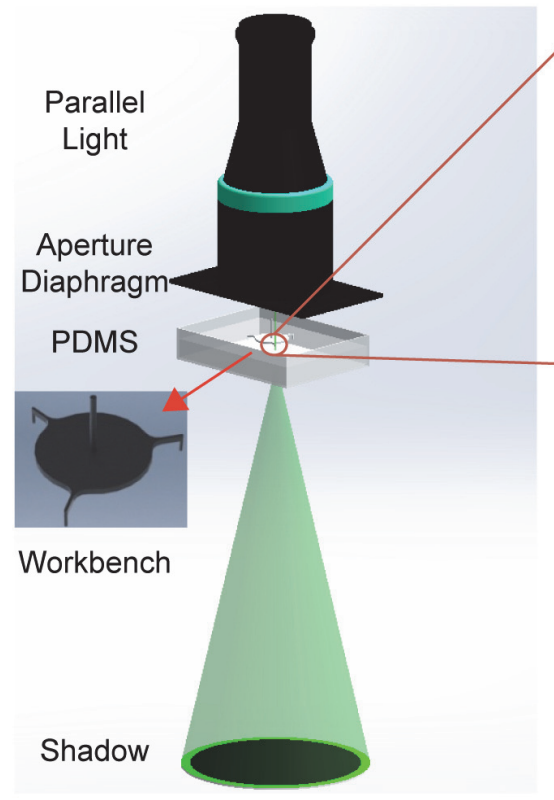

(a)

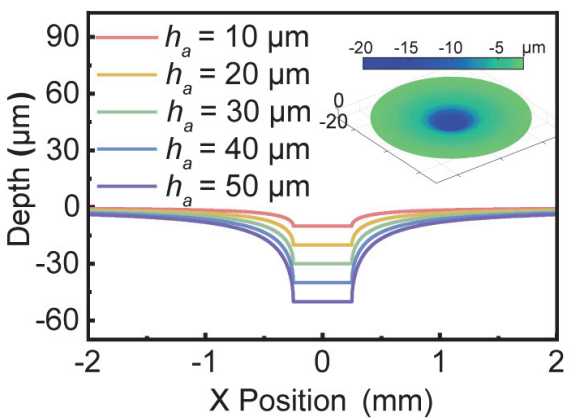

(e)

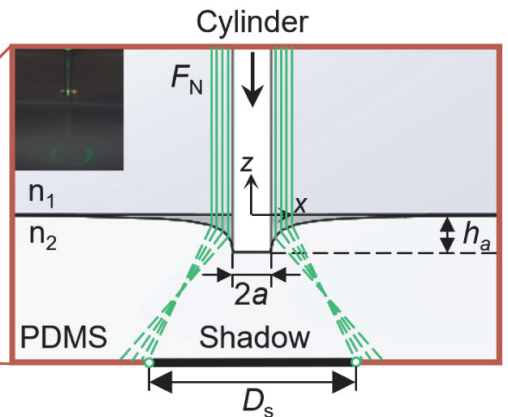

(b)

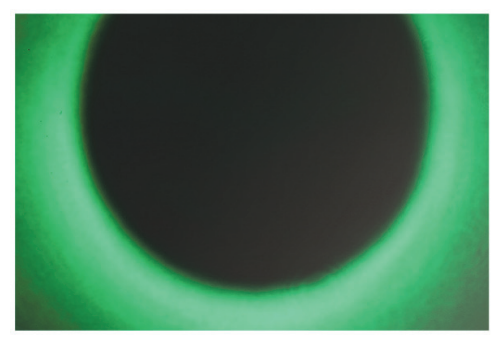

(c)

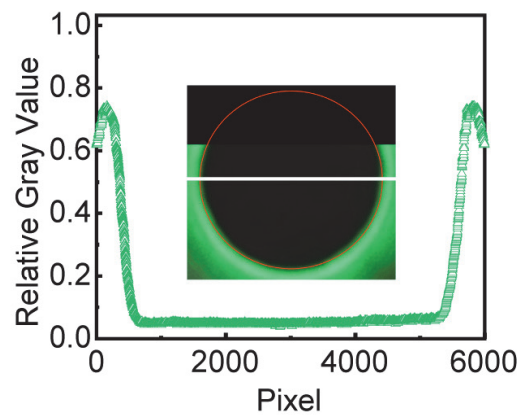

(f)

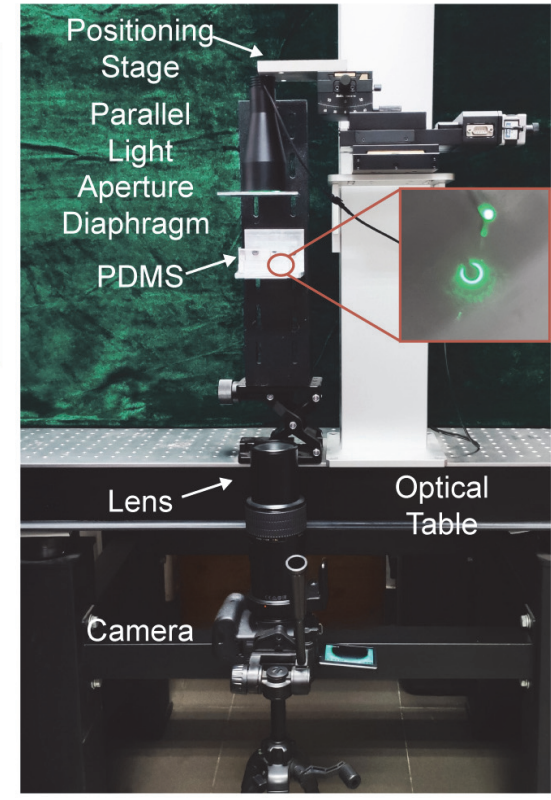

(d)

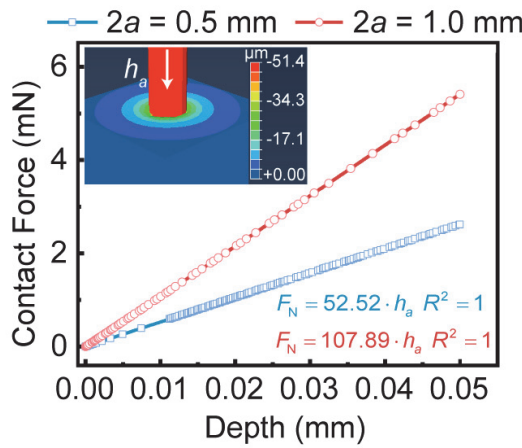

(g)

Fig. 1. Overview of VPMF sensor based on the shadow method. (a) Schematic of the overall structure. Inset: the diagram of the workbench. (b) Normal indentation by a cylindrical flat punch and sketch of light traveling of the sensor. Inset: the photograph of the deformed surface and light traveling. (c) Typical shadow photograph captured by the camera. Inset: the photograph of a shadow on a piece of white paper only for display. (d) The appearance of the VPMF sensor. Inset: the photograph of a shadow on a piece of white paper only for display. (e) Two-dimensional (2D) profiles of PDMS surface under different indentation depths with $2 a=0.5 \mathrm{~mm}$. Inset: three-dimensional (3D) illustration of depth profile. (f) Typical gray distribution of pixels in the horizontal diameter of a shadow. Inset: subpixel circle fitting of shadow (the white line represents the horizontal diameter). (g) Simulation of contact force between rigid cylindrical flat punch and PDMS elastic half-space. Inset: schematic of mechanics simulation.

extremely strict. However, disturbances are unavoidable effectively in many applications, such as the vibration of the vacuum chamber when a mechanics experiment is carried out in a vacuum environment [20] and the disturbances during the in-situ measurement at the industrial site [21].

In this paper, a novel bionic viscoelastic-polymer micro-force (VPMF) sensor based on the shadow method was proposed to measure micro force and displacement precisely with good linear performance. It was inspired by the phenomenon that the water strider walked on the water surface under sunlight and oval shadows, hundreds of times wider than the diameter of legs, were formed at the bottom of the pond due to the superhydrophobic legs [22]-[28]. The transparent viscoelastic polymer was used as the elastic sensitive element, whose damping property could effectively suppress environmental disturbances, improving the measurement precision [29]-[31]. The challenge of micro-force precise measurement is expected to be overcome by the VPMF sensor under the condition that the disturbance sources cannot be effectively isolated.

This paper is organized as follows. Section II introduces the overall design of the VPMF sensor. The optical simulation and the model of the shadow method are presented in Section III. The calibration experiments of force and displacement are presented in Section IV. Section V describes the experimental results of disturbance suppression and cantilever calibration. Section VI concludes this paper.

\section{OVERALl DESIGN}

In order to imitate the shadow which is hundreds of times larger than the diameter of the water strider legs, the VPMF sensor mainly consists of the parallel light source, the rigid cylindrical flat punch, and the transparent viscoelastic polymer [see Fig. 1(a)]. The involved basic mechanical principle and the principle of damping ratio to suppress disturbances are introduced. Details can be found in the following sections. 


\section{A. Configuration of the VPMF sensor}

The VPMF sensor [see Fig. 1 (d)] based on the shadow method was placed on an optical platform. A workbench (aluminum alloy, mass: $0.463 \mathrm{~g}$ ) with three rigid cylindrical legs (diameter $2 a=0.5 \mathrm{~mm}$ ) was located on the transparent viscoelastic polymer. The legs were distributed on the trisection points of a circle with a radius of $14 \mathrm{~mm}$. Polydimethylsiloxane (PDMS) is a transparent silicon-based crosslinked polymer, with adjustable functional groups and low-performance drift over time and temperature. It's suitable for micromechanical and chemical sensors, such as elastic materials used as accelerometers [32]. As a result, PDMS, Sylgard 184 (Dow Corning) served as the elastic element of the VPMF sensor, which was made by mixing base and curing agents at a ratio of 30: 1 (by weight). PDMS was vacuumed to remove bubbles and cured at $65^{\circ} \mathrm{C}$ for four hours. The size of PDMS was $60 \mathrm{~mm} \times$ $45 \mathrm{~mm} \times 10 \mathrm{~mm}$ with a smooth surface, whose boundary was fixed by a polycarbonate (PC) container.

A parallel light source (peak wavelength: $523 \mathrm{~nm}$, working distance: $300 \mathrm{~mm}$ ) was placed $50 \mathrm{~mm}$ above PDMS. The circular parallel light with a diameter of $0.7 \mathrm{~mm}$ vertically illuminated one of the rigid cylinders of the workbench through the aperture. The camera (resolution: $4000 \times 6000$, pixel size $S_{\mathrm{p}}$ $=3.72 \mu \mathrm{m}$, Canon EOS80D) was placed directly under PDMS to capture the photograph of the shadow. The working distance $H_{\mathrm{w}}$ was defined as the distance between the $5 \times$ zoom lens (MP-E 65mm f/2.8 1-5×) and the PDMS upper surface. The surface of PDMS was bent due to the downward movement of the workbench under the normal force $F_{\mathrm{N}}$ [see Fig. 1(b)]. The light refracted through the deformed interface, forming a shadow with a bright edge [see Fig. 1(c)]. The VPMF sensor detected the change of the shadow diameter and did a difference operation, thus dispensing with the use in a darkroom. The image gray distribution of the shadow is shown in Fig. 1(f). Firstly, the Sobel operator was used to detect the edge. Afterwards, the Zernike moment was used to locate the edge with sub-pixel accuracy. Finally, the shadow diameter was determined by the circle fitting.

\section{B. Damping ratio for noise suppression}

The system of the VPMF sensor can be regarded as the second-order system when there is a noise input. The vibration due to environmental disturbances is equivalent to white Gaussian noise with a power spectral density of constant $\mathrm{W}_{0}$. The frequency response of the system can be described as:

$$
H(\omega)=\frac{1}{k-m \omega^{2}+\mathrm{j} c \omega}
$$

where $m, k, c, \omega$ are equivalent mass, stiffness, damping coefficient of the system, and angular frequency respectively. The root mean square of the amplitude can be expressed as [33]:

$$
\overline{\mathrm{X}}^{2}=\frac{W_{0}}{2 \pi} \int_{-\infty}^{+\infty}|H(\omega)|^{2} d \omega=\frac{\mathrm{W}_{0} \omega_{n}}{4 \zeta k^{2}} .
$$

The noise amplification coefficient $\beta$ is defined as:

$$
\beta=\frac{\omega_{n}}{4 \zeta k^{2}}
$$

Where $\omega_{n}=\sqrt{k / m}$ is the natural frequency and $\zeta=c / 2 \sqrt{k m}$ is the damping ratio. The second-order system is a low-pass filter and the amplitude-frequency response decreases with the increase of the damping ratio. Hence, increasing the damping ratio can suppress the influence of noise within the natural frequency.

The free decay oscillation method [34] was applied to measure the damping ratio and natural frequency of the VPMF sensor. The rigid ball hit the workbench, and the change of the shadow diameter was captured by the high-speed camera (frame rate: 10000, MEMRECAM HX-6). The damping ratio of the VPMF sensor system was 0.22 and the natural frequency was $342.51 \mathrm{~Hz}$ after five repeated measurements. As shown in Fig. 2, the noise amplification coefficient is very low when the damping ratio is 0.22 .

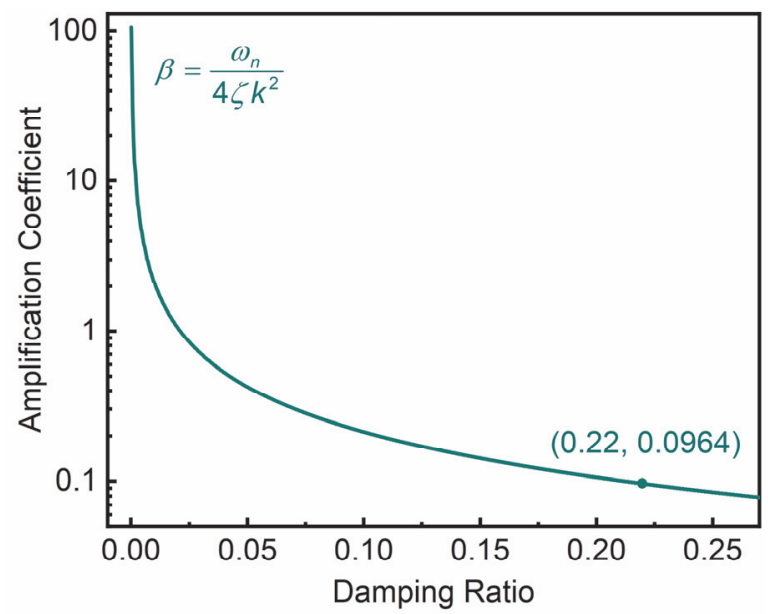

Fig. 2. The relationship between noise amplification coefficient $\beta$ and damping ratio $\zeta$.

\section{Normal contact of the cylindrical flat punch}

The rigid cylinders of the workbench were perpendicular to the surface of PDMS. It is equivalent to the contact of the rigid cylindrical flat punch with an elastic half-space. Explicit Solutions of force and displacements of the half-space surface for the cylindrical flat punch in the Johnson-Kendall-Roberts (JKR) model are [35]:

$$
\begin{gathered}
F_{\mathrm{N}}=\frac{2 a E}{1-v^{2}} \cdot h_{a}=k \cdot h_{a} \\
Z(x)=\frac{2 h_{a}}{\pi} \cdot \arcsin \left(\frac{a}{x}\right), x>a .
\end{gathered}
$$

The definition of parameters in this paper is summarized in Table I. Fig. 1(e) shows several profiles of PDMS outside the contact formed by the rigid cylinder with different depths. The simulation of contact force between the rigid cylinder and the elastic half-space [see Fig. 1(g)] indicated that the applied force was proportional to the indentation depth. Furthermore, the contact stiffness decreased with the diminution of cylinder diameter. Therefore, the sensitivity of the VPMF sensor can be improved by reducing the elastic modulus of PDMS and the diameter of the cylinder. 
TABLE I

DEFINITION OF PARAMETERS IN THE PAPER

\begin{tabular}{lll}
\hline \hline Symbol & \multicolumn{1}{c}{ Parameters } & Typical value \\
& & to $0.981 \mathrm{mN}$ \\
$F_{\mathrm{N}}$ & Normal force & $0.5 \mathrm{~mm}$ \\
$2 a$ & Diameter of the rigid cylinder & $10 \mathrm{~mm}$ \\
$H_{\mathrm{P}}$ & Thickness of PDMS & $0.09 \mathrm{Mpa}$ \\
$E$ & Elastic modulus of PDMS $(30: 1)$ & $0.4^{[38]}$ \\
$v$ & Poisson's ratio of PDMS $(30: 1)$ & $0-7 \mu \mathrm{m}$ \\
$h_{a}$ & Indentation depth of the workbench & $158.1 \mathrm{~N} / \mathrm{m}$ \\
$k$ & Contact stiffness & $\mathrm{N} / \mathrm{A}$ \\
$Z(x)$ & Displacements of PDMS outside the contact & $0.7 \mathrm{~mm}$ \\
$2 L$ & Diameter of parallel light & $<6000 \mathrm{pixels}$ \\
$D_{\mathrm{s}}$ & Shadow diameter & $<5^{\circ}$ \\
$\alpha$ & Angle of incidence of parallel light & $<5^{\circ}$ \\
$\beta$ & Angle of refraction of parallel light & $<5^{\circ}$ \\
$\varphi$ & Angle of emergence of parallel light & 1.4118 \\
$n$ & Refractive index of PDMS & $240 \mathrm{~mm}$ \\
$H_{\mathrm{w}}$ & Working distance & $3.72 \mu \mathrm{m}$ \\
$S_{\mathrm{p}}$ & Pixel size of the image sensor & 0.22 \\
$\zeta$ & Damping ratio & \\
\hline \hline
\end{tabular}

\section{Optical Simulation And Modeling}

In this section, the optical simulation of the shadow is completed to verify the relationship between the shadow diameter and the applied force. The model of the shadow method is derived and consistent with the simulation. The parameters are optimized by this model.

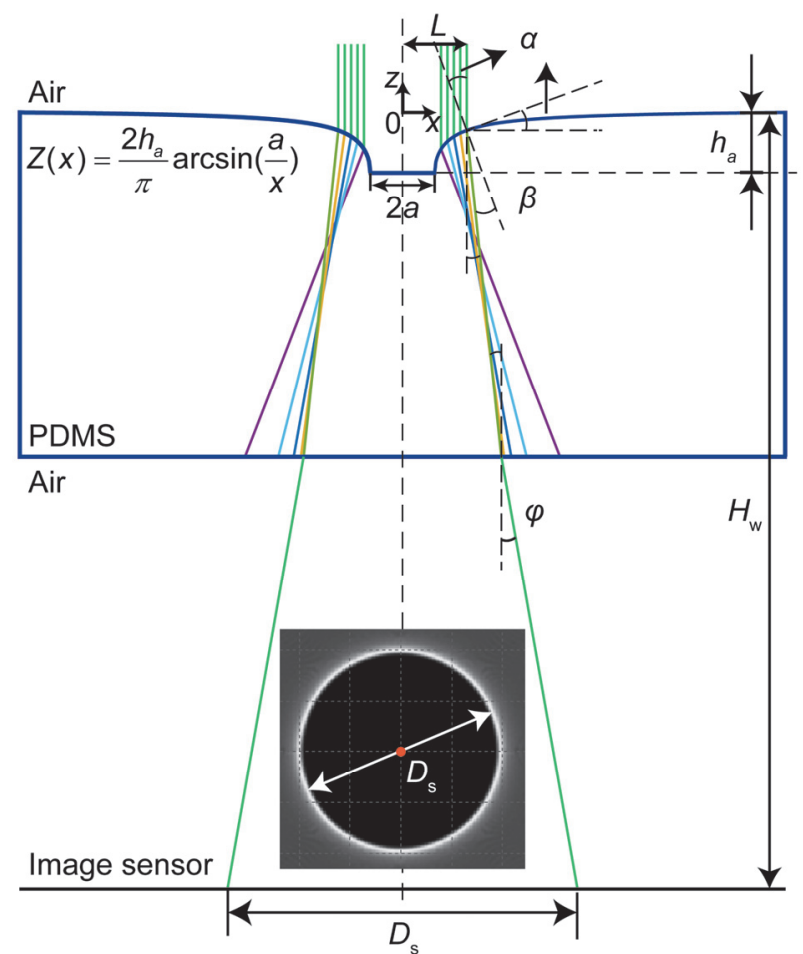

Fig. 3. The traveling of parallel light simulated numerically by Matlab. Inset: the diagram of the shadow simulated by TracePro.

\section{A. Optical simulation of the shadow}

Fig. 3 illustrates the traveling of parallel light through the deformed interface of PDMS by numerical simulation whose process is summarized as follows. The slope of a certain point on the profile outside the contact could be obtained according to (5) at a certain depth. The tangent of the incident angle of parallel light at this point was equal to the slope. Then the analytic expressions of each ray of refracted light and emergent light were able to be derived based on Snell's law at a certain depth. Finally, light emanating from the bottom of PDMS was captured by the image sensor of the camera. The shadow diameter could be calculated at this depth by the coordinates of the intersection of emergent light and the camera. The incident angles of the light near the contact were larger, so the refracted light had a larger deflection. The light away from the contact was opposite. As a result, the size of the shadow was determined by the outermost light when a certain depth was reached.

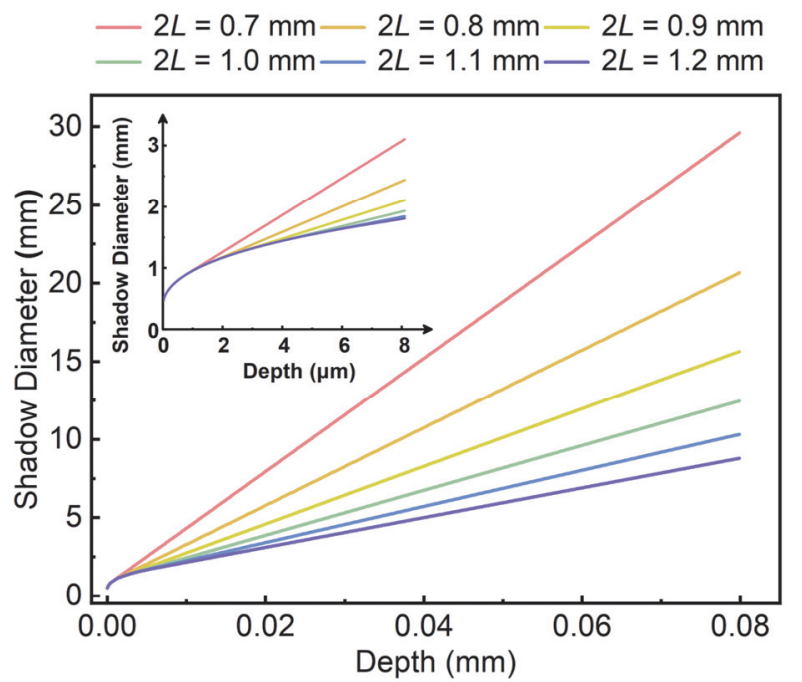

(a)

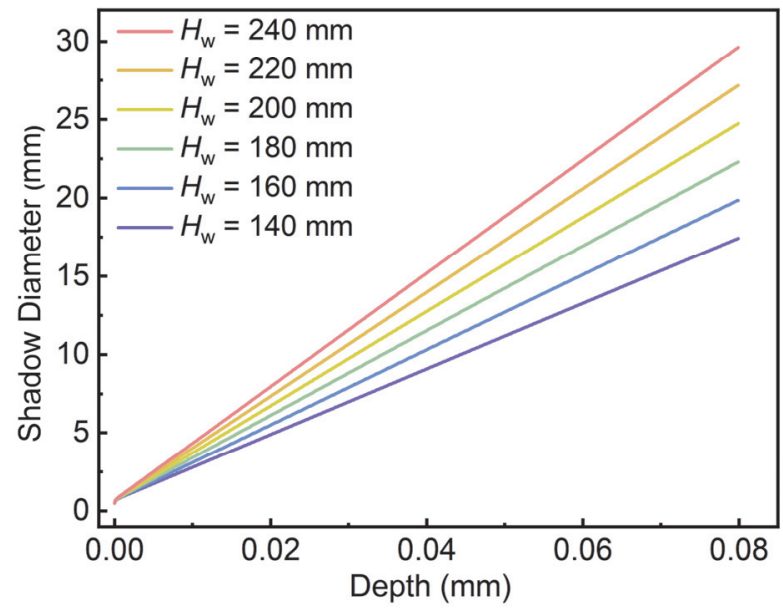

(b)

Fig. 4. Optical simulation of the shadow. (a) The relationship between the shadow diameter and indentation depth with different diameters of parallel light $\left(2 a=0.5 \mathrm{~mm}, H_{\mathrm{w}}=240 \mathrm{~mm}\right)$. Inset: the change of the shadow diameter is nonlinear with depth in that the light forming the shadow varies with the indentation depth from the inside out in the initial stage of contact. (b) The relationship between the shadow diameter and indentation depth with different working distances $(2 a=0.5 \mathrm{~mm}, 2 L=$ $0.7 \mathrm{~mm})$.

The formation of the shadow was also simulated by TracePro, where a shadow with a bright edge [see the inset of Fig. 3] could be obtained. The relationship between the indentation 
depth and the shadow diameter demonstrated by numerical analysis was quantitatively consistent with that by TracePro. The results of the shadow diameter in a good linear relationship with indentation depth are shown in Fig. 4(a) and (b). However, the shadow diameter was nonlinear with depth in the initial stage of contact. The reason was that the light forming the shadow varied with the indentation depth from the inside out. Only a few microns indentation depth was required to overcome the initial nonlinearity according to the simulation results when there was no significant difference between the diameter of parallel light and the cylinder diameter. Therefore, it's explicit that the VPMF sensor has good linear performance.

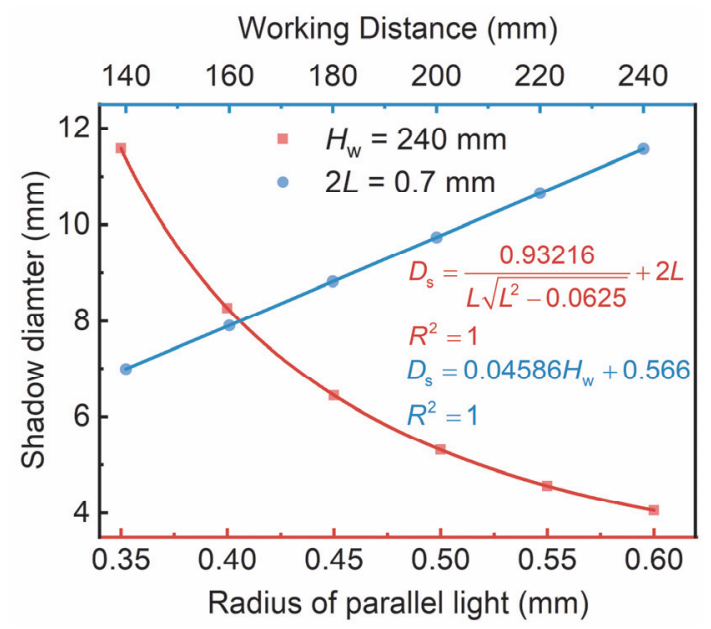

Fig. 5. The verification of the model of VPMF sensor. Red Line: the shadow diameter versus the diameter of parallel light $\left(2 a=0.5 \mathrm{~mm}, h_{a}=\right.$ $\left.30 \mu \mathrm{m}, H_{\mathrm{w}}=240 \mathrm{~mm}\right)$. Blue Line: the shadow diameter versus working distance $\left(2 a=0.5 \mathrm{~mm}, h_{a}=30 \mu \mathrm{m}, 2 L=0.7 \mathrm{~mm}\right)$.

\section{B. The model of the shadow method}

The incident angle $\alpha$ of the outermost light can be derived from (5) when parallel light illustrates the deformed surface of PDMS.

$$
\tan \alpha=\frac{d Z}{d x}(x=L)=\frac{2 a}{\pi L \sqrt{L^{2}-a^{2}}} \cdot h_{a}
$$

The relationship between the angles of emergence $\varphi$ and incidence $\alpha$ can be expressed as follows based on Snell's law under the condition of $\alpha<5^{\circ}$ :

$$
\begin{aligned}
& \frac{\tan \varphi}{\tan (\alpha-\beta)}=\frac{\tan \alpha}{\tan \beta}=n \\
& \tan \varphi=\frac{n(n-1) \tan \alpha}{n+\tan ^{2} \alpha}
\end{aligned}
$$

where $\beta, n$ are the angle of refraction and the refractive index of PDMS respectively. The formula $\tan \varphi \approx(n-1) \tan \alpha$ can be derived because $\tan ^{2} \alpha$ tends to be zero. Accordingly, the model between the shadow diameter and indentation depth is:

$$
\begin{aligned}
& \approx \frac{4 a(n-1) H_{\mathrm{w}}}{\pi L \sqrt{L^{2}-a^{2}}} \cdot h_{a}+2 L \\
\Delta D_{\mathrm{s}} & =\frac{4 a(n-1) H_{\mathrm{w}}}{\pi L \sqrt{L^{2}-a^{2}}} \cdot \Delta h_{a} .
\end{aligned}
$$$$
D_{\mathrm{s}}=2\left(L+H_{\mathrm{P}} \tan (\alpha-\beta)+\left(H_{\mathrm{w}}-H_{\mathrm{P}}\right) \tan \varphi\right)
$$

Hence, the model between normal force and the shadow diameter can be derived based on (9):

$$
F_{\mathrm{N}}=\frac{3 E}{1-v^{2}} \cdot \frac{\pi L \sqrt{L^{2}-a^{2}}}{2(n-1) H_{\mathrm{w}}} \cdot \Delta D_{\mathrm{s}} .
$$

It's explicit that the applied force is proportional to the change of the shadow diameter from the above derivation. The model was verified based on the above numerical simulation. The simulation results [see Fig. 5] indicated that the relationship between the shadow diameter and the diameter of parallel light, working distance conforms to (8). Therefore, the sensitivity of the VPMF sensor to force can be improved by reducing the diameter of parallel light and increasing working distance based on (9).

\section{Performance Test}

This section presents the calibration results of the force and displacement of the VPMF sensor. The equipment used in the tests is shown in Fig. 1(d). The sensitivity and the linear performance of the sensor are tested.
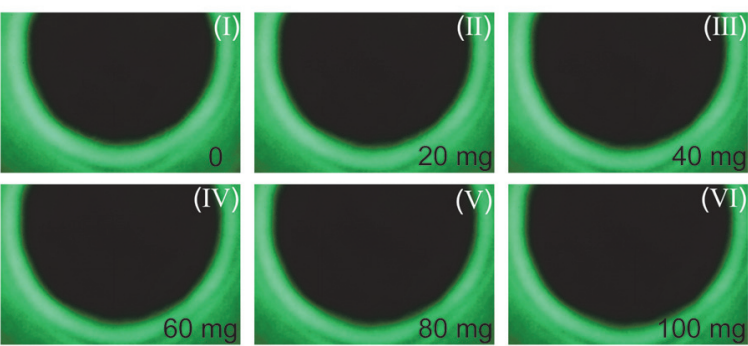

(a)

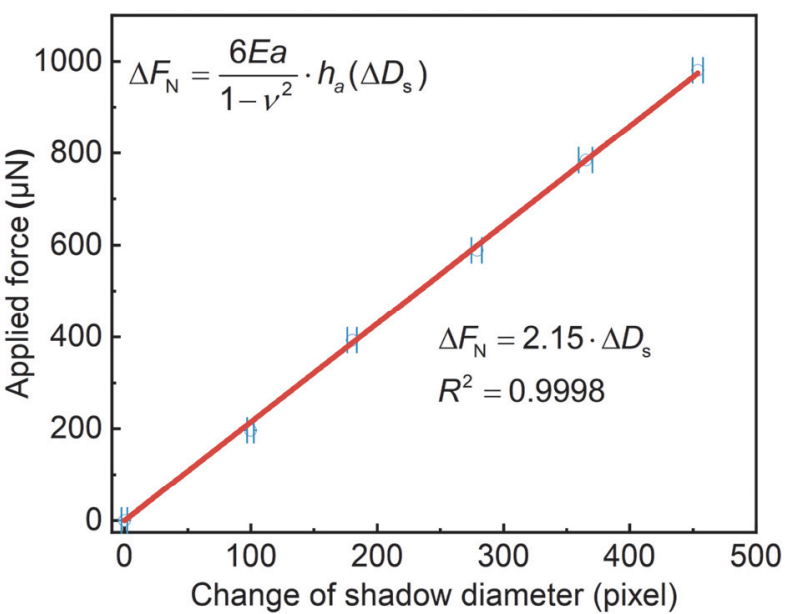

(b)

Fig. 6. The force calibration of the VPMF sensor. (a) The change of the shadow diameter with the number of mass blocks. (b) The linear fitting of applied force and the change of the shadow diameter with the goodness of $0.9998\left(2 L=0.7 \mathrm{~mm}, H_{\mathrm{w}}=240 \mathrm{~mm}\right)$. The slope of the line indicates the sensitivity of the sensor is $2.15 \mu \mathrm{N} /$ pixel.

\section{A. Force Calibration}

To achieve the best sensitivity of the VPMF sensor when the pixel size $\left(S_{\mathrm{p}}=3.72 \mu \mathrm{m}\right)$ is certain, the elastic modulus of viscoelastic polymer $\mathrm{E}$, the diameter of the rigid cylinder $2 a$ and the diameter of parallel light $2 L$ should be minimized, and the working distance $H_{\mathrm{w}}$ should be maximized on the ground of the shadow method. The ratio of base to cure of PDMS $=30: 1$ 
(by weight), $2 a=0.5 \mathrm{~mm}, 2 L=0.7 \mathrm{~mm}$ and $H_{\mathrm{w}}=240 \mathrm{~mm}$ were selected as the final parameters.

The initial incident angle due to the weight of the workbench was $3.03^{\circ}$, which met the derivation condition of $\alpha<5^{\circ}$. The 20 mg mass blocks measured precisely by an electronic balance (resolution: $0.01 \mathrm{mg}$, Sartorius Corp., ME235S) were prepared to calibrate the VPMF sensor. The camera was calibrated to correct the shadow image distortion. Capturing the shadow when the sensor unloaded was the beginning of calibration. Then the standard mass blocks were placed on the load button of the workbench one by one to capture the current image [see Fig. 6(a)] respectively. Repeated experiments were conducted five times. The relationship between applied force and the shadow diameter [see Fig. 6(b)] indicated that the sensitivity of the VCMF sensor was $2.15 \mu \mathrm{N} /$ pixel. Subpixel edge subdivision could further improve the resolution of the sensor. As the size of the camera's image sensor limited the maximum shadow diameter, the measurement range of the VPMF sensor was $0.981 \mathrm{mN}$. However, only considering the incident angle $\alpha$ $<5^{\circ}$ and the weight of the workbench, the linear measurement range could reach $2.96 \mathrm{mN}$ theoretically.

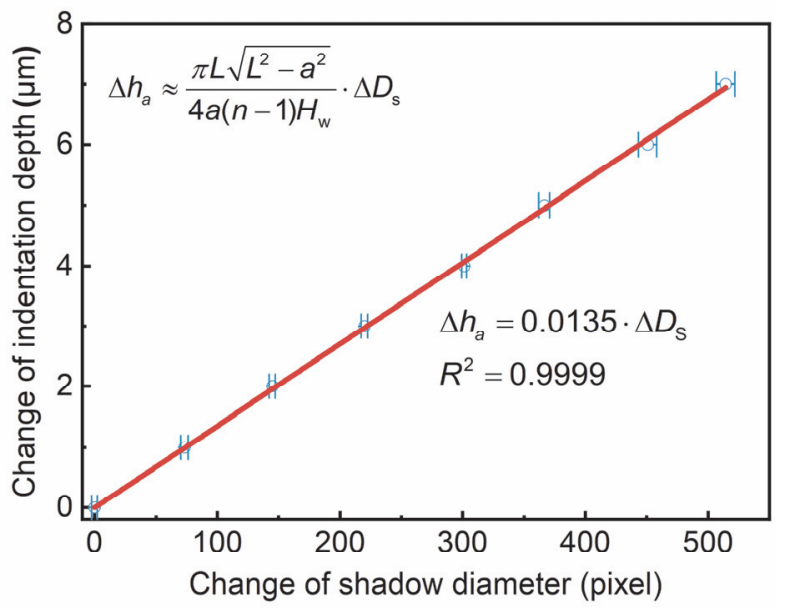

Fig. 7. The linear fitting of the indentation depth and the change of the shadow diameter with the goodness of $0.9999\left(2 L=0.7 \mathrm{~mm}, H_{\mathrm{w}}=240\right.$ $\mathrm{mm})$. The slope of the line indicates the displacement sensitivity of the sensor is $13.5 \mathrm{~nm} / \mathrm{pixel}$

\section{B. Displacement Calibration}

The parameters of the VPMF senor remained constant. Firstly, the vertical precision $\mathrm{z}$ positioner (resolution: $1 \mathrm{~nm}, \mathrm{PI}$, P-622.ZCD) was coupled with the workbench and stepped down at a fixed distance of $1 \mu \mathrm{m}$. Afterwards, the shadow photograph was captured every time the workbench moved down. The repeated experiments were conducted five times to obtain the relationship between indentation depth and the change of the shadow diameter (see Fig. 7). The results indicated that the displacement sensitivity of the VPMF sensor was $13.5 \mathrm{~nm} /$ pixel with a range of $7 \mu \mathrm{m}$. Subpixel edge subdivision could further improve the displacement resolution. Similarly, only considering the incident angle $\alpha<5^{\circ}$ and the weight of the workbench, the linear measurement range of the displacement could reach $18.59 \mu \mathrm{m}$ theoretically.

\section{EXPERIMENTAL RESULTS}

In order to verify the anti-disturbance ability of the VPMF sensor, the no-load experiment is carried out between the sensor and a cantilever beam with about the same stiffness. The output of the displacement (or force) represents the effect of environmental disturbances on them. The ability makes it suitable for calibrating the stiffness of cantilever beams. The vibration of cantilever beams to be tested has a bad influence on the calibration result of the stiffness. As a result, the sensor with the ability of anti-disturbance and measuring the force and displacement is needed.

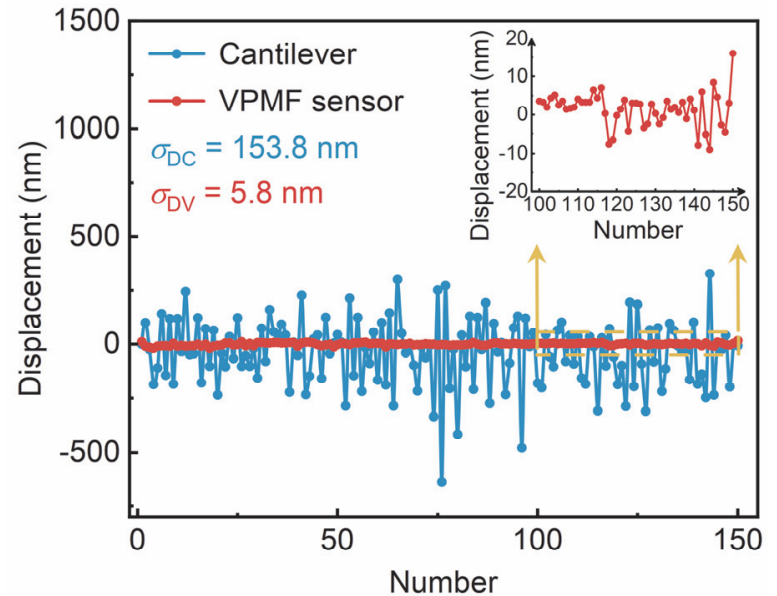

Fig. 8. The damping property suppressing the environmental disturbances. The red line and blue line: the displacement outputs of the VCMF sensor and the cantilever beam with the stiffness of $165.67 \mathrm{~N} / \mathrm{m}$ due to environmental disturbances.

\section{A. Disturbance suppression}

To visually demonstrate the suppression of system damping on disturbances, a cantilever beam with the stiffness of 165.67 $\mathrm{N} / \mathrm{m}$ was prepared to be compared with the VPMF sensor. The damping ratio and the natural frequency of this cantilever were 0.0002 and $28.57 \mathrm{~Hz}$ respectively, which were measured by the laser interferometer (resolution: $10 \mathrm{~nm}$, Renishaw RLE10) based on the free decay oscillation method. The no-load test of the VPMF sensor and the cantilever beam was carried out simultaneously in the absence of strict control of environmental disturbances. The displacement outputs of the VPMF sensor and the cantilever (see Fig. 8) were collected by camera and laser interferometer respectively at an interval of 2 seconds with a total of 150 data points. The repeatability of the VPMF sensor and cantilever was $5.8 \mathrm{~nm}$ (0.43 pixels) and $153.8 \mathrm{~nm}$ respectively. Thus, the damping property of PDMS can effectively suppress environmental disturbances. What's more, the sensor is able to be quickly stabilized to facilitate continuous measurement because of the damping property.

Compared with the above micro-force sensor based on the cantilever, the VPMF sensor reduced the influence of disturbances by about $96.23 \%$, thus improving the measurement precision. The damping ratio of the sensor can be further increased by polymer modification to suppress environmental disturbances [37], [38]. However, the VPMF sensor has a short life span, because the PDMS (Dow Corning, Sylgard 184) has a shelf life of only two years at room temperature. 


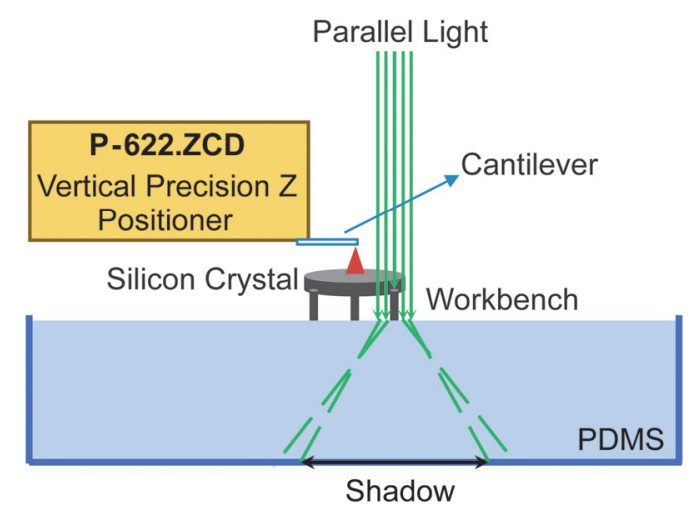

(a)

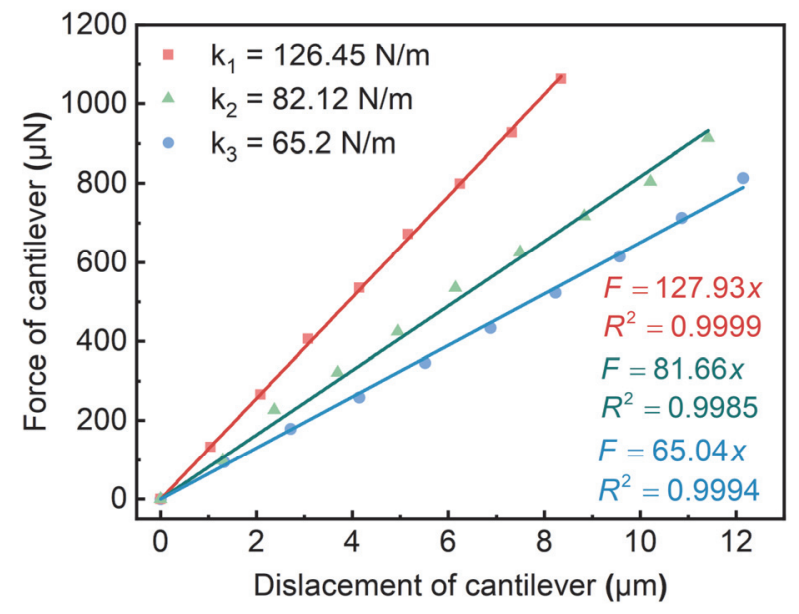

(b)

Fig. 9. The stiffness calibration of the cantilever based on the COC method. (a) The schematic illustration of the calibration. (b) The force versus the displacement of the cantilever beam under test. The stiffness values of the three cantilever beams were $126.45 \mathrm{~N} / \mathrm{m}, 82.12 \mathrm{~N} / \mathrm{m}$, and $65.2 \mathrm{~N} / \mathrm{m}$ based on the balance method. The slopes (unit: $\mathrm{N} / \mathrm{m}$ ) of the fitting curve were the calibration results.

\section{B. Calibrating the stiffness of cantilever beam}

The cantilever beam works as a widely used force sensor, whose spring constant must be calibrated before it can be used for quantitative force measurements. It is of great significance to the accuracy of the measurement results. The vibration of the cantilever beam to be calibrated due to environmental disturbances has an extremely bad effect on the precision of the stiffness calibration result. For example, the cantilever was calibrated based on the cantilever-on-cantilever (COC) method [39]. The VPMF sensor based on the shadow method can measure force and displacement with high precision simultaneously, and its damping property can effectively suppress environmental disturbances. As a result, the VPMF sensor (as the reference cantilever) is suitable for the cantilever stiffness calibration based on the COC method. The cantilever under test was fixed on the vertical precision $\mathrm{z}$ positioner to move down until contacting the load button of the workbench [see Fig. 9(a)]. The photograph of the shadow was captured at this time as the start point of calibration. The shadow photographs were captured one by one as the downward step displacements of the cantilever at a precise distance $Z$ of $2 \mu \mathrm{m}$, thus the force and displacement values of the cantilever were obtained. And the stiffness $k_{\mathrm{b}}$ can be calculated according to:

$$
k_{\mathrm{b}}=\frac{F_{\mathrm{N}}}{Z-h_{a}} .
$$

Compared to the calibration results based on the balance method [40] (by P-622.ZCD and ME235S), the stiffness values of the cantilevers calibrated by the two methods were consistent [see Fig. 9(b)].

The stiffness values of the reference cantilever and the cantilever under test should be matched (the ratio of stiffness should be between 0.3 and 3 ) to reduce the uncertainty during calibrating the cantilever based on the COC method [41]. The VPMF sensor can change the contact stiffness by adjusting the elastic modulus of polymer and the leg diameter of the workbench, so it's suitable for calibrating cantilevers with different stiffness.

\section{CONCLUSION}

In this paper, the bionic VPMF sensor based on the shadow method was presented. Parallel light illuminated the deformed surface of PDMS, and the refracted light formed a shadow with a bright edge at the bottom. The measured force was exactly proportional to the change of the shadow diameter. The sensitivity of the sensor was $2.15 \mu \mathrm{N} /$ pixel with a measurement range of $0.981 \mathrm{mN}$. The resolution could be further improved by subpixel edge subdivision. The damping ratio of the sensor was 0.22 , which could reduce the influence of disturbances by about $96.23 \%$ compared to the micro-force sensor based on the cantilever.

The viscoelastic polymer with better mechanical properties may further promote the development of the VPMF sensor as the rapid development of materials science. The VPMF sensor can be further miniaturized based on the shadow method. The potential application of the VPMF sensor is to reliably measure micro forces in complex environments. Meanwhile, it also has broad application prospects in the study of mechanical properties of materials and the examination of biological samples. In the future, we will develop a dynamic micro-force sensor with the help of a high-speed camera to measure the dynamic micro force, and improve the dynamic performance of the dynamic micro-force sensor by the damping property, such as reducing the response time and overshoot.

\section{REFERENCES}

[1] M. Armano et al., "Sub-Femto-g Free Fall for Space-Based Gravitational Wave Observatories: LISA Pathfinder Results," Phys Rev Lett, vol. 116, no. 23, p. 231101, Jun 102016.

[2] Q. Zhu, K. Vliet, N. Holten - Andersen, and A. Miserez, "A Double Layer Mechanochromic Hydrogel with Multidirectional Force Sensing and Encryption Capability," Advanced Functional Materials, vol. 29, no. 14, 2019.

[3] Y. Asai, Y. Yokokura, and K. Ohishi, "Fine Force Reproduction of Environmental Haptic Sensations Based on Momentum Control," IEEE Transactions on Industrial Electronics, vol. 63, no. 7, pp. 4304-4313, 2016.

[4] H. Guo et al., "Artificially innervated self-healing foams as synthetic piezo-impedance sensor skins," Nat Commun, vol. 11, no. 1, p. 5747, Nov 122020.

[5] H. Xie, X. Meng, H. Zhang, and L. Sun, "Development of a Magnetically Driven Microgripper for PicoNewton Force-Controlled Microscale 
Manipulation and Characterization," IEEE Transactions on Industrial Electronics, vol. 67, no. 3, pp. 2065-2075, 2020.

[6] T. Nozaki and H. Krebs, "Development of an Optical Sensor Capable of Measuring Distance, Tilt, and Contact Force," IEEE Transactions on Industrial Electronics, pp. 1-1, 2021.

[7] Y. Wu et al., "China's first step towards probing the expanding universe and the nature of gravity using a space borne gravitational wave antenna," Communications Physics, vol. 4, no. 1, 2021

[8] D. L. Hu, B. Chan and J. W. Bush, "The hydrodynamics of water strider locomotion," Nature, vol. 424, no. 6949, pp. 663-666, 2003

[9] J.-S. Koh et al. "Jumping on water: surface tension-dominated jumping of water striders and robotic insects," Science, vol. 349, no. 6247, pp. 517-521, 2015

[10] E. Yang, J. H. Son, S. I. Lee, P. G. Jablonski, and H. Y. Kim, "Water striders adjust leg movement speed to optimize takeoff velocity for their morphology," Nat Commun, vol. 7, p. 13698, Dec 72016.

[11] L. Persano et al., "High performance piezoelectric devices based on aligned arrays of nanofibers of poly(vinylidenefluoride-co-trifluoroethylene)," Nat Commun, vol. 4, p. $1633,2013$.

[12] A. A. M. Bui, A. V. Kashchuk, M. A. Balanant, T. A. Nieminen, H. Rubinsztein-Dunlop, and A. B. Stilgoe, "Calibration of force detection for arbitrarily shaped particles in optical tweezers," Sci Rep, vol. 8, no. 1, p 10798, Jul 172018

[13] N. Khatibzadeh et al., "Determination of motility forces on isolated chromosomes with laser tweezers," Sci Rep, vol. 4, p. 6866, Oct 312014.

[14] M. Zou et al., "Fiber-tip polymer clamped-beam probe for high-sensitivity nanoforce measurements," Light Sci Appl, vol. 10, no. 1, p. 171, Aug 272021.

[15] F. Orban, "Damping of materials and members in structures," Journal of Physics: Conference Series, vol. 268, 2011.

[16] Q. Li et al., "Measurements of the gravitational constant using two independent methods," Nature, vol. 560, no. 7720, pp. 582-588, Aug 2018.

[17] P. Sun, M. Zhao, J. Jiang, Y. Zheng, Y. Han, and L. Song, "The Differential Method for Force Measurement Based on Electrostatic Force," Journal of Sensors, vol. 2017, pp. 1-7, 2017.

[18] K. S. Kazimierski, I. Piotrowska-Kurczewski, F. Böhmermann, and O. Riemer, "A statistical filtering method for denoising of micro-force measurements," The International Journal of Advanced Manufacturing Technology, vol. 87, no. 5-8, pp. 1693-1704, 2016.

[19] Y. Sun et al., "Modeling Electromagnetic Force and Axial-Stiffness for an Electromagnetic Negative-Stiffness Spring Toward Vibration Isolation," IEEE Transactions on Magnetics, vol. 55, no. 3, pp. 1-10, 2019.

[20] A. Wang, H. Wu, H. Tang, Y. Liu, and X. Liang, "Development and testing of a new thrust stand for micro-thrust measurement in vacuum conditions," Vacuum, vol. 91, pp. 35-40, 2013.

[21] S. Li and K. Zhu, "In-situ tool wear area evaluation in micro milling with considering the influence of cutting force," Mechanical Systems and Signal Processing, vol. 161, 2021.

[22] H. Lu, Y. Zheng, J. Jiang, W. Yin, Y. Meng, and Y. Tian, "A Shadow-Based Nano Scale Precision Force Sensor," IEEE Sensors Journal, vol. 19, no. 6, pp. 2072-2078, 2019.

[23] Y. Zheng, H. Lu, W. Yin, D. Tao, L. Shi, and Y. Tian, "Elegant Shadow Making Tiny Force Visible for Water-Walking Arthropods and Updated Archimedes' Principle," Langmuir, vol. 32, no. 41, pp. 10522-10528, Oct 182016.

[24] H. Lu et al., "Propulsion Principles of Water Striders in Sculling Forward through Shadow Method," Journal of Bionic Engineering, vol. 15, no. 3 , pp. 516-525, 2018.

[25] Y. Zheng, W. Yin, H. Lu, and Y. Tian, "Revealing stepping forces in sub-mg tiny insect walking," Chinese Physics B, vol. 29, no. 12, 2020

[26] W. Yin, Y. L. Zheng, H. Y. Lu, X. J. Zhang, and Y. Tian, "Three-dimensional topographies of water surface dimples formed by superhydrophobic water strider legs," Applied Physics Letters, vol. 109 no. 16,2016

[27] Y. Zheng, H. Lu, J. Jiang, D. Tao, W. Yin, and Y. Tian, "Walking of spider on water surface studied from its leg shadows," Chinese Physics $B$, vol. 27 , no. 8,2018 .

[28] J. Z. Ma, H. Y. Lu, X. S. Li, and Y. Tian, "Interfacial phenomena of water striders on water surfaces: a review from biology to biomechanics," Zool Res, vol. 41, no. 3, pp. 231-246, May 182020.
[29] Z. Lou, S. Chen, L. Wang, K. Jiang, and G. Shen, "An ultra-sensitive and rapid response speed graphene pressure sensors for electronic skin and health monitoring," Nano Energy, vol. 23, pp. 7-14, 2016.

[30] I. K. Lin, Y.-M. Liao, Y. Liu, K.-S. Ou, K.-S. Chen, and X. Zhang, "Viscoelastic mechanical behavior of soft microcantilever-based force sensors," Applied Physics Letters, vol. 93, no. 25, 2008.

[31] P. Du, C. Cheng, H. Lu, and X. Zhang, "Investigation of Cellular Contraction Forces in the Frequency Domain Using a PDMS Micropillar-Based Force Transducer," Journal of Microelectromechanical Systems, vol. 22, no. 1, pp. 44-53, 2013.

[32] I. D. Johnston, D. K. McCluskey, C. K. L. Tan, and M. C. Tracey, "Mechanical characterization of bulk Sylgard 184 for microfluidics and microengineering," Journal of Micromechanics and Microengineering, vol. 24, no. 3, 2014.

[33] Y. Zheng et al., "Improving environmental noise suppression for micronewton force sensing based on electrostatic by injecting air damping," Rev Sci Instrum, vol. 85, no. 5, p. 055002, May 2014.

[34] J. Turunen et al., "Comparison of Three Electromechanical Oscillation Damping Estimation Methods," IEEE Transactions on Power Systems, vol. 26, no. 4, pp. 2398-2407, 2011.

[35] V.L. Popov, M. He $\beta$, E Willert, Handbook of contact mechanics: exact solutions of axisymmetric contact problems. Springer Nature, ch. 3, pp. 67-124, 2019.

[36] J. Chen, K. E. Wright, and M. A. Birch, "Nanoscale viscoelastic properties and adhesion of polydimethylsiloxane for tissue engineering," Acta Mechanica Sinica, vol. 30, no. 1, pp. 2-6, 2014.

[37] J. Zhao, N. Jiang, D. Zhang, B. He, and X. Chen, "Study on Optimization of Damping Performance and Damping Temperature Range of Silicone Rubber by Polyborosiloxane Gel," Polymers (Basel), vol. 12, no. 5, May 242020.

[38] B. Wang, A. G. Moura, J. Chen, A. Erturk, and Y. Hu, "Characterization of hydrogel structural damping," Extreme Mechanics Letters, vol. 40, 2020.

[39] M.-S. Kim, J.-H. Choi, J.-H. Kim, and Y.-K. Park, "Accurate determination of spring constant of atomic force microscope cantilevers and comparison with other methods," Measurement, vol. 43, no. 4, pp. $520-526,2010$

[40] M.-S. Kim, J.-H. Choi, J.-H. Kim, and Y.-K. Park, "SI-traceable determination of spring constants of various atomic force microscope cantilevers with a small uncertainty of $1 \%, "$ Measurement Science and Technology, vol. 18, no. 11, pp. 3351-3358, 2007.

[41] W. G. S. Gibson C T, Myhra S, "Determination of the spring constants of probes for force microscopy/spectroscopy," Nanotechnology, vol. 7, no. 3, p. $259,1996$.

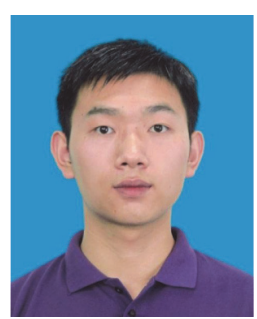

Yong Yang received the B.S. and M.S. degrees from Tianjin University (TJU), Tianjin, China, in 2017 and 2019, respectively.

$\mathrm{He}$ is currently working toward the Ph.D. degree in the State Key Laboratory of Precision Measuring Technology and Instruments, Tianjin University. His research interests include angle measurement and microforce measurement.

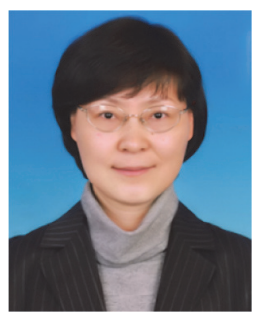

Meirong Zhao received the M.S. and Ph.D. degrees from Tianjin University (TJU), Tianjin, China, in 1993 and 1996, respectively.

She is currently a Professor with the State Key Laboratory of Precision Measuring Technology and Instruments, TJU. Her research interests include photoelectric measurement and control technology, visual inspection, and micro-nano measurement. 


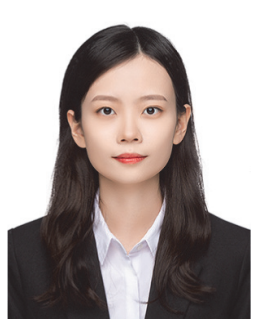

Dantong Li received the B.S. degree from Tianjin University, Tianjin, China, in 2020.

She is currently working toward the M.S. degree in the State Key Laboratory of Precision Measuring Technology and Instruments, Tianjin University. Her current research focuses on microforce measurement.

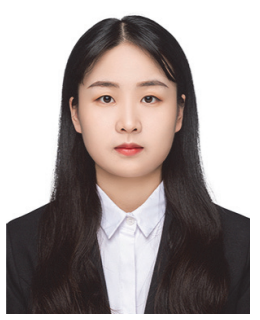

Moran Tao received the B.S. degree from Tianjin University, Tianjin, China, in 2020.

She is currently working toward the M.S. degree in the State Key Laboratory of Precision Measuring Technology and Instruments, Tianjin University. Her current research focuses on dynamic force measurement.

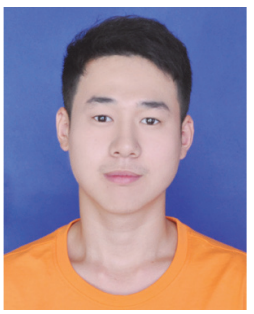

Chunyuan Zhu received the B.S. degree from China University of Geosciences, Wuhan, China, in 2020.

$\mathrm{He}$ is currently working toward the M.S. degree in the State Key Laboratory of Precision Measuring Technology and Instruments, Tianjin University. His current research focuses on micro-thrust measurement.

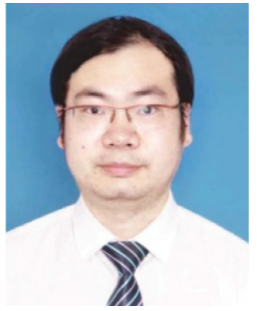

Yelong Zheng received the M.S. and Ph.D. degrees from Tianjin University (TJU), Tianjin, China, in 2012 and 2015, respectively.

$\mathrm{He}$ is currently an Assistant Professor with the State Key Laboratory of Precision Measuring Technology and Instruments, Tianjin University. His current research interests include microforce measurement and vivo sensitive measuring forces and tribology.

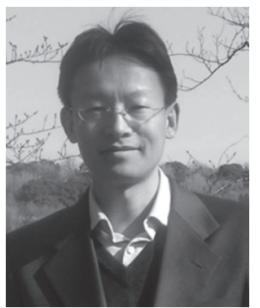

Yu Tian received the bachelor's and Ph.D. degrees in mechanical engineering from Tsinghua University in 1998 and 2002, respectively. Subsequently he joined the State Key Laboratory of Tribology. He is currently a Professor and the Vice Director of the State Key Laboratory of Tribology, Tsinghua University, China.

$\mathrm{He}$ held a post-doctoral position at the University of California at Santa Barbara, Santa Barbara, with Prof. Jacob Israelachvili (from 2005 to 2007), and Visiting Associate Professor at Nanyang Technology University for five months. His research interest is the science and technology at the interface of physics, materials, engineering, and biology to understand the physical laws of adhesion, friction, and rheology. He is the Editor-in-Chief of the Journal of Bio \& Tribo-Corrosion and an Editorial Board Member of Biotribology and Friction.

Dr. Tian has received the Wen Shizhu-Maple Award-Young Scholar Award in 2012, the Young Scholar Achievement Award of the Society of Mechanical Engineering of China in 2011, the Outstanding Young Scholar Award of the Chinese Tribology Institute in 2009, and the National Excellent Doctoral Dissertation of China in 2004. 